\title{
Abordagem sobre os aspectos gerais do corioangioma placentário: uma revisão narrativa
}

\author{
Approach to general aspects of placental chorioangioma: a narrative review
}

Aproximación a los aspectos generales del corioangioma placentario: una revisión narrativa

Carol Ferreira de Souza ${ }^{1 *}$, Ana Paula Oliveira Pinto ${ }^{1}$.

\section{RESUMO}

Objetivo: Discorrer sobre os tumores placentários. Revisão bibliográfica: O corioangioma placentário é um tumor que está pode ser constatado em cerca de uma das gestações e em sua grande maioria é caracterizado como assintomático. É um tumor benigno e de etiologia desconhecida, descrito na literatura que aqueles com diâmetro maior do que $4 \mathrm{~cm}$ e estão correlacionadas as complicações materno fetais. A real frequência desse tumor é difícil de saber, pois as massas pequenas não são detectadas durante 0 controle pré-natal, e o torna adequado é importante para a detecção oportuna de alterações durante a gravidez. Considerações finais: Muito importante o diagnóstico precoce sendo o método padrão ouro a ultrassonografia, preferencialmente com Doppler, e a ressonância magnética pode complementar as imagens ultrassonográficas. Os tratamentos são classificados como de suporte para manejo das complicações ou definitivo quando visa a eliminação do tumor. Existe a necessidade de realizar outros estudos afim de compreender melhor as maneiras de obter o diagnóstico precoce e desta maneira possibilitar uma intervenção que cessar o surgimento ou atenue as complicações relacionadas a esta patologia, resultando em desfechos gestacionais favoráveis.

Palavras-chave: Hemangioma, Neoplasia, Placenta.

\begin{abstract}
Objective: To discuss placental tumors. Bibliographic review: Placental chorioangioma is a tumor that can be seen in about one of the pregnancies and the vast majority is characterized as asymptomatic. It is a benign tumor of unknown etiology, described in the literature that those with a diameter greater than $4 \mathrm{~cm}$ are correlated with maternal and fetal complications. The actual frequency of this tumor is difficult to know, as small masses are not detected during prenatal control, and making it suitable is important for the timely detection of changes during pregnancy. Final considerations: Early diagnosis is very important and the gold standard method is ultrasound, preferably with Doppler, and magnetic resonance imaging can complement ultrasound images. Treatments are classified as supportive for the management of complications or definitive when aiming to eliminate the tumor. There is a need to carry out other studies in order to better understand the ways to obtain an early diagnosis and thus enable an intervention that stops the onset or attenuates the complications related to this pathology, resulting in favorable gestational outcomes.
\end{abstract}

Keywords: Hemangioma, Neoplasm, Placenta.

${ }^{1}$ Hospital Universitário Getúlio Vargas - Universidade Federal do Amazonas (UFAM), Manaus - AM.
*E-mail: carolcfs21@gmail.com

SUBMETIDO EM: 1/2022

ACEITO EM: 1/2022

PUBLICADO EM: 2/2022 


\section{RESUMEN}

Objetivo: Discutir los tumores placentarios. Revisión bibliográfica: El corioangioma placentario es un tumor que se puede observar en aproximadamente uno de los embarazos y la gran mayoría se caracteriza por ser asintomático. Es un tumor benigno de etiología desconocida, descrito en la literatura que aquellos con un diámetro mayor a $4 \mathrm{~cm}$ se correlacionan con complicaciones maternas y fetales. La frecuencia real de este tumor es difícil de saber, ya que no se detectan masas pequeñas durante el control prenatal, y su adecuación es importante para la detección oportuna de cambios durante el embarazo. Consideraciones finales: El diagnóstico precoz es muy importante y el método estándar de oro es la ecografía, preferentemente con Doppler, y la resonancia magnética puede complementar a las ecografías. Los tratamientos se clasifican en de soporte para el manejo de las complicaciones o definitivos cuando se pretende eliminar el tumor. Existe la necesidad de realizar otros estudios con el fin de comprender mejor las formas de obtener un diagnóstico precoz y así posibilitar una intervención que detenga la aparición o atenúe las complicaciones relacionadas con esta patología, resultando en resultados gestacionales favorables.

Palabras clave: Hemangioma, Neoplasma, Placenta.

\section{INTRODUÇÃO}

Historicamente, é possível afirmar que a placenta é um órgão que desperta curiosidade científica há muitos anos, tanto por sua formação quanto por sua função. $O$ termo placenta foi originalmente derivado do grego deutera, mais tarde conhecido como secundina, uma palavra de origem latina. No entanto, o primeiro a chamá-lo corretamente de placenta foi o grego, plakoûta, que significa bolo redondo. O nome do órgão foi usado pela primeira vez por Phalopius em 1523 e descrito em "De Re Anatomica" por Realdus Columbus, e assim, portanto, o termo foi aceito por anatomistas e obstetras (CARINA FG, et al., 2015)

As doenças neoplásicas primárias da placenta abrangem uma ampla gama de situações que são rotuladas em dois grupos principais: doenças trofoblásticas e não trofoblásticas, sendo que as doenças não trofoblásticas ocorrem com maior frequência, sempre com cursos benignos (ABDALLA N, et al., 2014).

Enquanto a Doença Trofoblástica Gestacional (DTG) conglomera um grupoheterogêneo de proliferação celular gerada a partir do epitélio trofoblástico placentário, com formatos clínicas benignas, caracterizadas pela Mola Hidatiforme Completa (MHC) e Parcial (MHP) e por desenhos malignos, representados pela mola invasora, coriocarcinoma, tumor trofoblástico do sítio placentário e tumor trofoblástico epitelioide, ajuntados sob o epíteto de Neoplasia Trofoblástica Gestacional (NTG) (TSE KY e NGAN HY, 2012).

O corioangioma é o tumor vascular não trofoblástico mais comum da placenta, com incidência estimada em $1 \%$, e embora sua etiologia exata não esteja totalmente esclarecida, acredita-se que seja resultado da proliferação anormal de vasos sanguíneos em diferentes estágios de diferenciação. Estroma fibroso originário do tecido coriônico (LIM FY, et al., 2015), e ainda é descrito como tumor benigno não trofoblástico mais frequente da placenta, com incidência estimada de 0,6\% (WILLIS C, et al., 2019)

Os tumores são nomeados com base em sua histogênese e histopatologia. Para tumores benignos, a regra é adicionar o sufixo "oma" (tumor) aos termos que especificam o tecido de onde se originaram. (MINISTÉRIO DA SAÚDE, 2013). Embora a maioria dos corioangiomas placentários sejam assintomáticos, eles podem estar associados a complicações maternas ou fetais significativas, como polidrâmnio, oligoidrâmnio, trabalho de parto prematuro, descolamento prematuro da placenta, pré-eclâmpsia, restrição de crescimento fetal (FGR), anemia fetal, insuficiência cardíaca congestiva, fetal não imune hidropsia e até morte fetal (ZANARDINI C, et al., 2010)

Mais comumente, os corioangiomas são pequenos com significância clínica insignificante (VENTILADOR M; SKUPSKI DW, 2014) As complicações estão associadas a corioangiomas gigantes $(>4 \mathrm{~cm}) \mathrm{A}$ vascularização aumentada do corioangioma placentário tem sido associada a um risco aumentado de complicações.Corioangiomas gigantes $(>5 \mathrm{~cm})$ apresentam consequências mais graves para 0 feto; 0 tamanho do tumor mostrou estar correlacionado com piores resultados perinatais devido à 
insuficiência cardíaca de alto débito devido ao sequestro de sangue, incluindo cardiomegalia com disfunção cardíaca, polidrâmnio, anemia fetal, hidropisia fetal e restrição de crescimento fetal (RFG) (FAN M e MOOTABAR H, 2015).

Dependendo do tamanho, vascularidade e localização do corioangioma, uma pequena parte deles pode resultar em complicações materno-fetais graves. As complicações comuns incluem polidrâmnio, préeclâmpsia, ruptura prematura da membrana, descolamento prematuro da placenta, trabalho de parto prematuro, restrição de crescimento fetal (FGR), hidropisia fetal não imune, anemia e até morte fetal. $O$ diagnóstico precoce e o acompanhamento regular podem indicar ou melhorar o prognóstico da mãe / feto. Já o exame pré-natal de perto, principalmente por ultrassom, deve ser praticado rotineiramente. Os tumores pequenos geralmente são monitorados a cada três ou quatro semanas, enquanto os tumores grandes requerem exames a cada uma ou duas semanas (AKERCAN F, et al., 2012)

O tamanho da massa, presença de hidropisia e idade gestacional na ocorrência da insuficiência cardíaca tem sido relatada como os principais determinantes do resultado perinatal em gestações complicadas por corioangioma 5,6. A maioria dos corioangiomas são pequenas lesões assintomáticas que são encontradas apenas no pós-natal após o corte cuidadoso da placenta5. Por outro lado, grandes tumores têm sido associados a uma infinidade de resultados perinatais adversos, incluindo restrição de crescimento, parto prematuro (PTB), morte intrauterina (DIU) e resultado anormal do neuro (IACOVELLA C, et al., 2014)

O corioangioma placentário gigante está associado a uma variedade de complicações obstétricas. A mortalidade perinatal estimada varia de 30 a $40 \%$. Atualmente, os corioangiomas com repercussão fetal podem ser tratados por diversos métodos. No entanto, é importante estabelecer conduta expectante (acompanhamento rigoroso por ultrassom), para planejamento do momento do parto, ou tratamento conservador (se a idade gestacional permitir), mesmo optando por tratamento invasivo. Outras opções incluem: redução em caso de sintomas maternos devido à hiperfiltração (RAMIREZ-CARDENAS A, et al., 2018).

A ultrassonografia desempenha um papel importante no diagnóstico pré-natal do corioangioma placentário. Além disso, é útil monitorar a circulação fetal e prever o resultado fetal usando fluxo em cores e Doppler pulsado durante o acompanhamento. Como esse tumor vascular benigno leva a complicações tão variadas ainda precisa ser totalmente elucidado (LIU H, et al., 2014).

Se as complicações se desenvolverem no final da gravidez, o parto planejado é uma opção razoável. No entanto, a maioria das complicações ocorre no início da gestação, tornando o parto prematuro iatrogênico inapropriado devido ao alto risco de mortalidade e morbidade secundária à prematuridade (BUCA D, et al., 2020)

As características ultrassonográficas do corioangioma placentário incluem uma massa sólida hipoecóica e heterogênea, que se projeta para a cavidade amniótica na superfície fetal da placenta. A avaliação ultrassonográfica Doppler do corioangioma é a chave na caracterização da vascularização do tumor, o que confirma o diagnóstico em contraste com outras massas sólidas (JAUNIAUX E e OGLE R, 2000).

O mesmo autor discorre que as características ultrassonográficas do corioangioma placentário incluem uma massa sólida hipoecóica e heterogênea, que se projeta para a cavidade amniótica na superfície fetal da placenta. A avaliação ultrassonográfica Doppler do corioangioma é a chave na caracterização da vascularização do tumor, o que confirma o diagnóstico em contraste com outras massas sólidas (JAUNIAUX E e OGLE R, 2000). Na Ressonância Magnética (RM), o corioangioma parece isointenso à placenta nas imagens ponderadas em $\mathrm{T} 1 \mathrm{e}$ tem sinal variável, geralmente sendo hiperintenso à placenta nas imagens ponderadas em T2. Em casos de corioangiomas grandes e muito heterogêneos, a ressonância magnética pode ser útil para diferenciar os corioangiomas de outras massas placentárias raras, como os teratomas (JHA P, et al., 2016).

A aplicação do Doppler colorido permite a visualização dos vasos de alimentação entrando na massa placentária e da vascularização difusa peritumoral. Nos casos mais graves, podem coexistir com o tumor sinais de insuficiência cardíaca de alto débito, incluindo cardiomegalia, polidrâmnio, aumento da velocidade na Artéria Cerebral Média (ACM) e hidropisia fetal (LIU H, et al., 2014). 
Devido à raridade dos casos e à diversidade de suas características clínicas, nenhum consenso foi ainda estabelecido sobre o manejo de tumores placentários não trofoblásticos (ABDALLA N, et al., 2017). Já a Sociedade Internacional de Ultrassom em Obstetrícia e Ginecologia (ISUOG) recomenda a localização da placenta, sua relação com o orifício cervical interno, em caso de anormalidade no exame de rotina. Descreva sua aparência para identificar achados anormais, incluindo hemorragias, cistos múltiplos e massas placentárias, como corioangioma (SALOMON LJ, et al., 2011)

Se surgirem complicações no terceiro trimestre, o parto planejado é uma opção razoável. No entanto, a maioria das complicações ocorrem no início da gravidez, e o parto prematuro não é adequado para o parto prematuro iatrogênico devido à alta mortalidade e morbidade secundária ao parto prematuro. Mais estudos são necessários para elucidar melhorias no tratamento fetal resultado dessas gestações e determinar as consequências a longo prazo das manifestações cardiovasculares e do neurodesenvolvimento desses fetos com comprometimento hemodinâmico persistente induzido por tumor (BUCA D, et al., 2020)

E ainda Segundo Buca D, et al (2020) o diagnóstico pré-natal do corioangioma placentário é baseado na visualização de uma massa placentária hipoecóica, arredondada e bem circunscrita, localizada na superfície fetal da placenta, com arquitetura homogênea ou heterogênea.

Diante do que já foi descrito sobre os tumores placentários e entendendo a grande importância do assunto abordado, o objetivo do presente trabalho foi de realizar uma revisão bibliográfica e assim discorrer sobreos coriangiomas a fim de entender melhor e assim elucidar melhor sobre as características clínicas, diagnóstico, evolução e tratamento.

\section{REVISÃO BIBLIOGRÁFICA}

O corioangioma placentário foi descrito pela primeira vez por ultrassonografia por Asokan $\mathrm{S}$, et al. (1978). Sabe-se que é um corioangioma, hemangioma, mixoma, hemangioma ou fibroma e pode consistir em vasos sanguíneos fetais conectados por tecido conjuntivo (ASOKAN S, et al., 1978).

As lesões vasculares da placenta consistem em um grupo distinto, mas inter-relacionado de entidades, como corioangioma, aneurisma de artéria coronária, aneurisma coronário focal e aneurisma coronário multifocal difuso. Hemangioma placentário com origem na camada coriônica (WU Z e HU W, 2016). É uma lesão nodular vascular dilatada composta por capilares, estroma e trofoblastos, mais comum entre a $32^{a} \mathrm{e}$ $37^{\text {a }}$ semanas de gestação e em placentas gemelares e pré-eclâmpticas. (PEC) (PEREIRA NRGP, et al., 2011)

O corioangioma placentário é caracterizado por alterações ao nível arterial e venoso, devido a uma proliferação exagerada das estruturas vasculares ao nível do endotélio e das vilosidades coriónicas, que geram uma localização na margem placentária ou junto à placa. (PÉREZ GEG, et al., 2013)

É considerada uma malformação arteriovenosa; na maioria dos casos é única, embora também possa ser múltipla ou mesmo dispersa, o que é uma raça muito rara. A aparência macroscópica característica desse tumor é redonda ou oval, encapsulada, com margens bem definidas, consistência dura ou mole e coloração marrom-avermelhada e afirmaram que o corioangioma costuma ser solitário e raramente múltiplo. Sua forma é regular, redonda ou oval. Eles geralmente são de cor marrom-avermelhada e de aparência carnuda, e podem ser macios ou firmes em consistência. Histologicamente, apareceu um conjunto de vilosidades placentárias médias e terminais muito dilatadas, que não estavam separadas por uma cápsula fibrosa, mas por um conjunto de vilosidades (ÁLVAREZ C, et al., 2007).

Embora possam ocorrer sem complicações, a importância clínica do corioangioma reside na amplitude e gravidade das possíveis complicações obstétricas e neonatais, que vão desde pré-eclâmpsia, líquido amniótico e parto prematuro, até anemia, insuficiência cardíaca congestiva, edema, restrição de crescimento intrauterino e feto morte, acredita-se que a etiologia desse fenômeno se deva à presença de anastomose vascular fetoplacentária, ao sequestro de hemácias e plaquetas fetais pela massa tumoral e as consequentes alterações cinéticas e cardiovasculares do fluxo sanguíneo (SEPULVEDA W, et al., 2003). 
Considerada uma lesão incomum de acordo com Punales SJ (2018), e esse tumor foi relatado em 1\% das placentas ao exame microscópico e em aproximadamente 1:3.500 a 1:9.000 neonatos em evidência clínica.

No estudo de Soto AL, et al. (2015), apresentamos uma paciente do sexo feminino, 25 anos, primípara, grupo sanguíneo $0+$, com teste de anticorpos irregular e sem histórico de interesse pessoal. A triagem ultrassonográfica no primeiro trimestre é satisfatória e o risco de aneuploidia é baixo. Morfologia ultrassonográfica de segundo trimestre com 21,4 semanas, feto do sexo masculino, presença de polidrâmnio, veias umbilicais dilatadas e derrame pericárdico ecocardiográfico e regurgitação tricúspide. Ao estudo ultrassonográfico, as glândulas placentárias eram impressionantes com 110 na parte superior A 29 $\mathrm{mm}$ imagem hipoecogênica da extremidade da superfície fetal a placenta, altamente vascularizada, com fluxo de baixa resistência, compatível com corioangioma placentário gigante.

Dada a gravidade desses achados e o impacto hemodinâmico do corioangioma no coração fetal, o acompanhamento semanal foi determinado pela conclusão do estudo por amniocentese genética com cariótipo $46 \mathrm{XY}$ normal e sorologia, materna e fluida adicional amniótico, que também foram negativos. $\mathrm{O}$ controle semanal continua, embora na semana 24,4 de gestação, o diagnóstico de natimorto é os controles semanais continuaram apesar do diagnóstico confirmado de natimorto com 24,4 semanas de gestação, indução médica do trabalho de parto com prostaglandinas, que exigiu parto artificial e curetagem, sem outros eventos clínicos. O estudo anatomopatológico descreve um feto de $500 \mathrm{~g}$ com morfometria correspondente a 24 semanas de gestação sem malformações externas e sem alterações histológicas associadas em órgãos internos, com exceção do coração, que apresenta cavidade mais dilatada do que o esperado morfometricamente (SOTO AL, et al., 2015).

Já no estudo de Zacharis K, et al. (2020) discorre sobre uma paciente em sua primeira gestação caucasiana de 29 anos de idade apresentando um histórico médico normal mas que apresentou em exame de rotina às 21 semanas de gestação apresentando massa placentária medindo $1,8 \times 2,2 \mathrm{~cm}$, sendo realizada uma avaliação ultrassonográfica detalhada revelou uma lesão circunscrita, hipoecóica, com vascularização aumentada, projetando-se para a cavidade amniótica; assim, foi feito um diagnóstico preliminar de corioangioma placentário e agendada uma vigilância pré-natal rigorosa.

Os autores acima citaram que o feto cresceu adequadamente, a velocidade sistólica máxima da artéria cerebral média, bem como o índice de líquido amniótico estavam dentro dos limites normais, à medida que a gravidez avançava. Com 34 semanas e 6 dias de gestação, a massa na placenta aumentou de tamanho para 6,27 x 5,38 cm. paciente apresentou-se ao nosso serviço de emergência queixando-se de movimentos fetais reduzidos com 37 semanas de gestação onde a paciente solicitou parto por cesariana, A placenta foi encaminhada para exame anatomopatológico que confirmou o diagnostic (ZACHARIS K, et al., 2020)

Rondon-Tapia M, et al. (2017), em seu relato de caso discutem o caso de um corioangioma placentário de crescimento rápido em uma paciente primípara de 20 anos sem complicações de qualquer tipo, até a realização e avaliação por ultrassonografia. Com 22 semanas de idade, a placenta implantada na face anterior do útero era visível desta vez, apresentando um tumor placentário bem vascularizado de 4,7 × 3,2 $\mathrm{cm}$, sem pedículo vascular e o feto crescendo normalmente. Às 28 semanas, a ultrassonografia em escala de cinza mostrou uma massa clara e levemente ecogênica $(9,9 \times 7,1 \mathrm{~cm})$ no lado esquerdo da placenta.

Liu $\mathrm{H}$, et al. (2014) descreveram que $\mathrm{O}$ uso da ultrassonografia possibilita o diagnóstico e 0 acompanhamento antes do parto, o que facilita o monitoramento fetal rigoroso e permite 0 uso de ferramentas para auxiliar na tomada de decisões adequadas. A ultrassonografia em escala de cinza revelou uma massa ecogênica complexa que estava bem estabelecida do resto da placenta, e o tumor se projetava na cavidade amniótica, geralmente próximo à inserção do cordão umbilical.

Corroborando sobre o tema descrito Desai T e VishwanaTH U (2020) destacam o caso de uma paciente de 26 anos que foi encaminhada para trabalho de parto prematuro com 31 semanas mais 5 dias em sua primeira gravidez. E na mesma vertente em seu relato de caso Perreira NRGB, et al. (2011) destacaram em 
seu relato de caso a importância da placenta como órgão fundamental durante a gravidez e sua relevância na avaliação ultrassonográfica pré-natal, permitindo o diagnóstico precoce de anormalidades e condições anormais: a capacidade de ajustar o monitor e resultados fetais.

No estudo de Pérez GEG, et al. (2013) os autores relataram que o aneurisma de artéria coronária, um tumor benigno com hiperplasia de capilares e células do estroma viloso, está associado a gestações múltiplas, pré-eclâmpsia e defeitos congênitos fetais. Este artigo relata o caso de uma gestante de 38 anos (29 semanas de gestação). Teve 11 gestações, 9 partos e 1 aborto. Durante a avaliação perinatal, a paciente apresentou polidrâmnio grave e sofrimento fetal agudo. O exame histopatológico da placenta revelou uma massa com áreas de hemorragia. $O$ exame microscópico revelou pequena hiperplasia capilar fetal e imunocoloração positiva para CD34. Estudos histopatológicos de tumores primários não trofoblásticos devem distinguir a doença vascular coronariana de outras entidades, como hemangiomatose coronariana e coriocarcinoma, e o estudo deve relatar a presença de displasia mesenquimal por estar associada a doenças genéticas. Esse tumor está associado ao processo hipóxico feto-placentário e deve ser avaliado em pacientes com histórico de restrição de crescimento fetal, pré-eclâmpsia e gravidez múltipla.

Em seu relato de caso Willis C, et al. (2019) uma paciente multigestacional de 27 anos de idade foi notada em um exame de crescimento de rotina às 27 semanas de gestação para ter uma área hipoecoica placentária central medindo 6,7 ×6,0 ×4,4 cm. Uma ressonância magnética subsequente confirmou uma massa sólida na placenta anterior; portanto, foi feito um diagnóstico preliminar de corioangioma placentário gigante. A repetição da ultrassonografia com 30 semanas de gestação indicou que a massa havia aumentado, com presença de polidrâmnio. A paciente apresentou movimentos fetais reduzidos com 31 semanas de gestação, e o parto foi por meio de cesariana de emergência. A placenta revelou um grande corioangioma

O tratamento do corioangioma permanece controverso. Opções de tratamento estão disponíveis, como drenagem do excesso de líquido amniótico e intervenções no próprio corioangioma, incluindo coagulação a laser e eletrocirurgia (BARROS A, et al., 2011).

Torres-Correa JE, et al. (2020) apresentaram, o caso de um jovem de 21 anos paciente sem controle pré-natal prévio, com corioangioma placentário diagnosticado com 26 semanas de gestação que formou lobo acessório de $7 \mathrm{~cm}$, com coloração positiva na análise imuno-histoquímica para anticorpos antimúsculo liso e CD34 e negativo para Ki67. O corioangioma placentário desencadeou complicações durante a gravidez, com parto prematuro, hidropisia fetal e crescimento intrauterine retardamento que leva à morte fetal. A falta de controle pré-natal em nossa paciente impediu a detecção precoce de anormalidades placentárias com desfecho fatal para o feto.

Ainda sobre este estudo de caso foi evidenciado que controle pré-natal adequado com monitoramento ultrassonográfico pode evitar eventos fatais, como aquele apresentado. A análise macroscópica e microscópica da placenta pode ajudar identificar casos e contribuir para as estatísticas de incidência e mortalidade (TORRES-CORREA JE, et al., 2020)

Diferentes intervenções têm sido propostas para melhorar os resultados perinatais. $O$ tratamento conservador baseia-se na monitorização ultrassonográfica e avaliação com Doppler Fetoplacentário. 0 tratamento invasivo é controverso devido às complicações associadas. $O$ uso de amniodrenagem tem sido proposto para aliviar os polidrâmnios, e as transfusões de sangue intrauterino estão sendo usadas atualmente para tratar a anemia fetal pelos efeitos do corioangioma (LIU H, et al., 2014).

Dada a associação entre corioangioma placentário e complicações perinatais, o acompanhamento prénatal rigoroso deve ser realizado regularmente. Se acontecerem complicações no final da gravidez, o parto deve ser avaliado, dependendo da maturidade fetal e do suporte neonatal disponível. No entanto, gestações com complicações mais graves geralmente ocorrem no final do segundo trimestre, momento em que o parto não é a opção preferida devido à prematuridade (RONDON-TAPIA M, et al., 2017). 


\section{CONSIDERAÇÕES FINAIS}

O corioangioma placentário pode acometer em aproximadamente $1 \%$ das placentas examinadas na histopatologia e é o tumor placentário benigno mais comum. O diagnóstico precoce visa reduzir a progressão tumoral e consequentes complicações maternas e fetais. É importante e a ultrassonografia é o método padrão ouro, preferencialmente o Doppler, e a ressonância magnética pode complementar as imagens de ultrassom. Portanto, ressalta-se a importância do diagnóstico precoce para que intervenções possam ser realizadas para prevenir ou amenizar as complicações associadas a essa patologia, resultando em bons desfechos gestacionais e que faz-se necessário realizar mais estudos sobre os coriangiomas a fim de entender melhor as características clínicas, diagnóstico, evolução e qual o melhor tratamento.

\section{REFERÊNCIAS}

1. ABDALLA N, et al. O ultrassom pode ser útil na seleção de métodos de tratamento ideais para gestações complicadas por tumores placentários não trofpblásticos? J Ultrason, 2017; 17(69): 116-122.

2. ABDALLA N, et al. Placental tumor (chorioangioma) as a cause of polyhydramnios: a case report. International Journal of Women's Health, 2014: 6.

3. AKERCAN F, et al. Insuficiência cardíaca de alto débito em feto com displasia tanatofórica associada a corioangioma placentário volumoso: relato de caso. J Clin Ultrasound, 2012; 40: 231.

4. BARROS A, et al. Corioangioma placentário gigante: uma causa rara de hidropisia fetal. Representante de Caso BMJ 2011; 2011: bcr0220113880.

5. BUCA D, et al. Resultado perinatal de gestações complicadas por corioangioma placentário: revisão sistemática e metanálise. Ultrassom Obstet Gynecol, 2020; 55: 441-449.

6. CARINA FG, et al. Desenvolvimento placentário e interações materno fetais na espécie equine. RPCV, 2015; 110: 593-594: $1-5 p$.

7. CZERESNI Ricardo Mamber, et al. Mapeamento Doppler Tridimensional de Corioangioma Placentário Antes de Ablação Fetoscópica a Laser. J Ultrasound Med, 2021; 9999:1-3.

8. FAN M, MOOTABAR H. Um raro corioangioma placentário gigante com evolução favorável: Relato de caso e revisão da literatura. J Clin Ultrassom. 2015; 43(4): 254-256.

9. IACOVELLA C, et al. Tumores vasculares fetais e placentários: o estado hiperdinâmico fetal persistente predispõe a um pior resultado do desenvolvimento neurológico a longo prazo. Ultrassom Obstet Gynecol 2014; 43: 658-661.

10. JHA P, et al. Imagem multimodalidade de massas placentárias: uma revisão pictórica. Abdom Radiol (NY), 2016; 41(12): 2435-2444.

11. LIM FY, et al. Corioangiomas gigantes: resultados perinatais e técnicas de desvascularização fetoscópica. Diagnóstico Fetal Ter 2015; 37: 18-23.

12. LIU $\mathrm{H}$, et al. História natural e resultado da gravidez em pacientes com corioangioma placentário. J Clin Ultrassom, 2014;42(2):74-80.

13. MANCUSO A, et al. Large placental chorioangioma. Acta Obstet Gynecol Scand, 2001; 80: 965-6.

14. PEREIRA NRGP, et al. Lesão vascular da placenta condicionando RCIU e hidropisia fetal não imune em gestação gemelar. Rev Braś Ginecol Obstét, 2011; 33(12): 421-5.

15. MINISTÉRIO DA SAÚDE. Secretaria de Atenção à Saúde, Departamento de Regulação. Avaliação e Controle, Coordenação Geral de Sistemas de InformaçãO. Brasília - DF: Brasil, 2013. Disponível em: https://bvsms.saude.gov.br/bvs/publicacoes/inca/manual_oncologia_14edicao.pdf.Acessado em 06 de janeiro de 2022.

16. PÉREZ GEG, et al. Corangioma placentário: abordagem clínicopatológica de um caso descrito na Colômbia. Gine col Obstet Mex, 2013;81:109-114.

17. PERREIRA NRGB, et al. Lesão vascular da placenta condicionando RCIU e hidropisia fetal não imune em gestação gemelar. Rev. Bras. Ginecol. Obstet, 2011; 33(12): 421-425.

18. PUÑALES SJ, et al. Corioangioma gigante de la placenta con buenos resultados perinatales. Revista Cubana de Obstetricia y Ginecología, 2018; 44(2).

19. RAMIREZ-CARDENAS, A.Corioangioma placentário gigante. Reporte de un caso. Ginecol. obstet. Méx. [online], 2018:86(10): 692-698.

20. RONDON-TAPIA M, et al. Corioangioma placentário gigante. Relatório de caso. Revista Peruana de Ginecología y Obstetricia, 2017; 62(4): 455-458

21. SALOMON LJ, et al. Comitê de Padrões Clínicos da ISUOG. Diretrizes práticas para a realização da ultrassonografia fetal de rotina no meio do trimestre. Ultrassom Obstet Gynecol, 2011; 37: 116-126.

22. SEPULVEDA W, et al. Perinatal outcome after prenatal diagnosis of placental chorioangioma. Obstet Gynecol, 2003; 102(5): 1028-33.

23. SOTO AL, et al. Corioangioma gigante placentario. Prog Obstet Ginecol. 2015.

24. TORRES-CORREA JE, et al. Corioangioma placentario: reporte de un caso en la Unidad de Patología del Hospital San Juan de Dios E.S.E. Rionegro-Antioquia. Medicina y Laboratorio, v. 24, Número 4, 2020.

25. TSE KY, NGAN HY. Gestational trophoblastic disease. Best Pract Res Clin Obstet Gynaecol, 2012;26(3): 357-70.

26. VENTILADOR M, SKUPSKI DW. Corioangioma placentário: revisão de literatura. J Perinat Med. 2014; 42(3): $273-9$.

27. WILLIS C, et al. Corioangioma placentário associado a polidrâmnio e hidropisia fetal. BMJ Case Rep. 2019; 12(1): e227828.

28. WU Z, HU W. Análise clínica de 26 pacientes com corioangiomas placentários histologicamente comprovados. Eur J Obstet Gynecol Reprod Biol. 2016; 199: 156-63.

29. ZACHARIS K, et al. Um caso raro de corioangioma placentário gigante com evolução favorável, PAMJ, 2020; 36(214).

30. ZANARDINI C, et al. Corioangioma placentário gigante: história natural e evolução da gravidez. Ultrasound Obstet Gynecol, 2010; 35: 332. 